\title{
Tratamiento en domicilio de trombosis venosa profunda con heparinas de bajo peso molecular. Experiencia de un año
}

\author{
C. G. SUÁREZ ÁLVAREZ, J. GARCÍA CAÑETE, M. D. HERRERO MENDOZA, \\ T. M. BELLVER ÁLVAREZ, R. ARBOIRO PINEL
}

Unidad de Hospitalización a Domicilio. Clínica Ntra. Sra. Concepción. Fundación Jimé nez Díaz. Madrid

TREATMENT OF DEEP VEIN THROMBOSIS WITH LOW MOLECU LAR WEIGHT HEPARINS AT HOME. ONE YEAR EXPERIENCE

\begin{abstract}
RESUMEN
La eficacia y seguridad de las heparinas de bajo peso molecular permiten que los pacientes con trombosis venosa profunda puedan tratarse en unidades de hospitalización a domicilio. Entre las heparinas de bajo peso molecular, la tinzaparina se administra en dosis única diaria facilitando el cumplimiento terapéutico.

Se revisan los veinte pacientes diagnosticados de trombosis venosa profunda ingresados en la unidad de hospitalización a domicilio de la Clínica de Nuestra Señora de la Concepción de Madrid entre diciembre de 1999 y diciembre de 2000. La edad media de los pacientes fue de $71+$ 15. Los factores de riesgo más frecuentes fueron cirugía en los tres meses previos $(19 \%)$, presencia de tumores $(15 \%)$ y trombosis previa $(15 \%)$.

Dieciocho pacientes recibieron tratamiento con tinzaparina. Ninguno presentó complicaciones ni efectos adversos.

El tratamiento con heparinas de bajo peso molecular y en particular con tinzaparina, es un tratamiento seguro y eficaz para la trombosis venosa profunda en nuestra unidad.
\end{abstract}

PALABRAS CLAVE: Trombosis venosa profunda. Heparinas bajo peso molecular. Tinzaparina. Tratamiento en domicilio.

\begin{abstract}
Treatment of patients with proximal vein thrombosis with low mole cular weight heparins is effective and safe. So it allows "hospital at home" care. Among low molecular weight heparins tinzaparin is given once daily, making the compliance easier.

The twenty patients with deep vein thrombosis who were assisted in the "hospital at home" unit of the Clínica Nuestra Señora de la Concep ción in Madrid from December 1999 to December 2000. The mean of age was $71+15$. The most frequent risk factors were surgery in past three months (19\%), known tumour (15\%) and previous venous thrombo sis $(15 \%)$.

Eighteen patients were treated with tinzaparin. No patients showed adverse effects nor complications.

Low molecular weight heparins, specially tinzaparin, are a safe and effective treatment for deep vein thrombosis in our unit.
\end{abstract}

KEY WORDS: Deep-vein trombosis. Low-molecular-weight heparins. Tinzaparin. Home-care.

Suárez Álvarez CG, García Cañete J, Herrero Mendoza MD, Bellver Álvarez TM, Arboiro Pinel R. Tratamiento en domicilio de trombosis venosa profunda con heparinas de bajo peso molecular. Experiencia de un año. An Med Interna (Madrid) 2003; 20: 134-136.

\section{INTRODUCCIÓN}

Las heparinas de bajo peso molecular han demostrado ser al menos tan seguras y eficaces como la heparina no fraccionada en el tratamiento y prevención de la trombosis venosa profunda (1-9). Tienen, además, las ventajas frente a ésta, de poderse administrar por vía subcutánea y de no precisar monitorización de laboratorio ya que su respuesta es más predecible por su mejor biodisponibilidad, mayor duración de la vida media y aclaramiento independiente de la dosis administrada. Por estas características, los pacientes con este diagnóstico son subsidiarios de recibir tratamiento en su domicilio, obteniéndose, en los estudios que comparan pacientes tratados en el hospital con heparina no fraccionada con pacientes tratados en su domicilio, resultados similares en cuanto a efectividad y seguridad $(1,2,4,5)$.

La tinzaparina es una heparina de bajo peso molecular (4.500), con un índice anti-Xa/anti-IIa de 1,9, obtenida desde la heparina por digestión con heparinasa; que, por sus características farmacocinéticas, puede administrarse una sóla vez al día para el tratamiento de la trombosis venosa profunda $(6,10)$. Esto hace que sea un fármaco muy emplea-

Trabajo aceptado: 20 de diciembre de 2001

Correspondencia: María Dolores Herrero Mendoza. Unidad de Hospitalización a Domicilio. Clínica Ntra. Sra. de la Concepción. Fundación Jiménez Díaz. Avda. Reyes Católicos, 2. 28040 Madrid. 
do en la Unidad de Hospitalización a Domicilio, ya que la dosis única favorece el cumplimiento de los pacientes y el que pueda ser administrado por personal sanitario de la Unidad teniendo la seguridad de que la administración ha sido correcta en aquellos casos de pacientes (y cuidadores) mayores y/o enfermos.

Se han revisado todos los pacientes diagnosticados de trombosis venosa profunda y tratados durante un año en la Unidad de Hospitalización a Domicilio de la Clínica de Ntra. Sra. de la Concepción (Fundación Jiménez Díaz), recogiéndose los datos relativos a las características de los pacientes, patología concomitante, tratamiento anticoagulante, evolución y existencia de complicaciones.

\section{CASOS APORTADOS}

Se presentan datos de los 20 pacientes diagnosticados de trombosis venosa profunda que ingresaron en la Unidad de Hospitalización a Domicilio entre diciembre de 1999 y diciembre de 2000. Los pacientes presentaban edades comprendiadas entre 33 y 95 años con una media de $71+15$ (mediana $=70)$. La distribución por sexos fue de 10 varones y 10 mujeres.

Entre los antecedentes personales que se recogieron los más frecuentes fueron los antecedentes quirúrgicos, en siete casos; y la existencia de un antecedente tumoral en 3 pacientes.

En el $25 \%$ de los pacientes, no se encontró factor de riesgo previo para enfermedad tromboembólica; cuando sí se encontraron, los más frecuentes fueron: cirugía en los tres meses previos a la enfermedad (19\%), tumores $(15 \%)$ y existencia de trombosis previa $(15 \%)$. En un solo un caso se encontró la existencia de Factor V de Leyden (Tabla I).

TABLA I

FACTORES DE RIESGO PARA EL DESARROLLO DE TVP

\begin{tabular}{lcc}
\hline Factor de riesgo & $\mathrm{n}$ & $\%$ \\
\hline Ninguno & 5 & 25 \\
Cirugía tres meses previos & 4 & 20 \\
Tumor & 3 & 15 \\
Trombosis previa & 3 & 15 \\
Traumatismo & 1 & 5 \\
Inmovilización crónica & 1 & 5 \\
Factor V Leyden & 1 & 5 \\
O besidad & 1 & 5 \\
Inmovilización transitoria (viaje) & 1 & 5 \\
\hline
\end{tabular}

Diecisiete pacientes $(85 \%)$ ingresaron directamente en la unidad de hospitalización a domicilio, frente a tres pacientes que, previo a su traslado a la unidad, habían estado ingresados en servicios de hospitalización convencial.

En 12 pacientes el tratamiento anticoagulante previo al traslado a su domicilio fue diferente de tinzaparina: en un caso consistió en heparina intravenosa, en el resto se emplearon otras heparinas de bajo peso molecular: enoxaparina en 7 casos y nadroparina en 4 casos. Posteriormente, al ser trasladados a su domicilio a cargo de la unidad; 6 de los pacientes tratados con enoxaparina, 3 de los que recibieron nadroparina y la paciente que había iniciado la anticoagulación con heparina intravenosa, recibieron tinzaparina hasta que se ajustó la dosis precisa de sintrom. En los 8 pacientes restantes se empleó tinzaparina desde el inicio. Por tanto, dieciocho pacientes en total recibieron tinzaparina.

En 2 pacientes se mantuvo al alta de la Unidad el tratamiento con heparina de bajo peso molecular mientras que en el resto se introdujeron anticoagulantes orales (acenocumarol) manteniéndose simultáneamente el tratamiento con heparina hasta que se ajustaba la dosis de sintrom necesaria para obtener un INR entre 2 y 3 , este período de tiempo fue de 6 días de media.

Ninguno de los pacientes presentó efectos adversos en relación con el tratamiento, ni complicaciones embólicas, ni recurrencias de la enfermedad durante el tiempo del estudio ni en los tres meses siguientes a su finalización.

En 9 pacientes de los 18 en los que se disponía de este dato, la heparina de bajo peso molecular era administrada por personal de la unidad, en cinco por el propio paciente y en los 4 restantes por familiares de éste.

\section{DISCUSIÓN}

La introducción de las heparinas de bajo peso molecular simplificó el tratamiento de la trombosis venosa profunda permitiendo que estos pacientes pudieran ser tratados en su domicilio con igual efectividad y seguridad que los pacientes tratados en el hospital con heparina no fraccionada $(1,2,4)$. Este tipo de hospitalización ofrece ventajas para el paciente en cuanto al entorno en el que se desarrolla el ingreso, permite una mejor educación sanitaria y disminuye la ocupación de camas hospitalarias. Permitiendo un seguimiento de la enfermedad y la posibilidad de vigilar la aparición de complicaciones así como un ajuste adecuado del tratamiento anticoagulante. La revisión de los pacientes ingresados durante un año en la Unidad de Hospitalización a domicilio apoya la seguridad y eficacia del tratamiento de estos pacientes con heparinas de bajo peso molecular sin necesidad de ingreso hospitalario convencional.

Desde que se dispuso de tinzaparina se eligió este fármaco para el tratamiento de la trombosis venosa profunda por su dosificación única diaria, lo que facilitaba el cumplimiento terapéutico en todos los pacientes, y reducía el número de visitas en aquéllos que necesitaban que el fármaco fuera administrado por personal sanitario. También la presentación comercial de este fármaco, en jeringas precargadas, facilita tanto su auto-adminsitración como la administración por parte de familiares.

Llama la atención la ausencia de complicaciones y de efectos adversos, que puede estar en relación con el pequeño tamaño de la muestra, pero que también concuerda con resultados obtenidos en otros estudios previos $(1,2,4)$.

En nuestro centro, por tanto, el tratamiento de la trombosis venosa profunda con tinzaparina en la unidad de hospitalización a domicilio es un tratamiento de elección. Esto permite valorar la posibilidad de tratamiento del tromboembolismo pulmonar, para el que ya se ha reconocido el empleo de tinzaparina $(3,10,11)$, también en esta unidad. 


\section{Bibliografía}

1. Levine M, Gent M, Hirsh J, Leclerc J, Anderson D, Weitz J, et al. A comparison of low-molecular-weight heparin administered primarily at home with unfractionated heparin administered in th hospital for proximal deep-vein thrombosis. N Engl J Med 1996; 334: 677-81.

2. Koopman MMW, Prandoni P, Piovella F, Ockelford PA, Brandjes DPM, van der Meer J, et al. Treatment of venous thrombosis with intravenous unfractionated heparin administered in the hospital as compared with subcutaneous low-molecular-weight-heparin administered at home. N Engl J Med 1996; 334: 682-7.

3. Weitz JI. (editor: Wood AJJ). Low-molecular-weight heparins. N Engl J Med 1997; 337: 688-98.

4. Harrison L, et al. Assessment of outpatient treatment of deep-vein thrombosis with low- molecular-weight heparin. Arch Intern Med 1998; 158: 2001-3.

5. Leibowitz RS. Deep vein thrombosis: Thinking inside out. Arch Intern Med 1998; 158: 1964.
6. Hedner U. Development of tinzaparin: a heparinase-digested low-molecular-weight heparin. Semin Thromb Hemost 2000; 26 (Supl. 1): 23-9.

7. Martineau P, Tawil N. Low-molecular-weight heparins in the treatment of deep-vein thrombosis. Ann Pharmacother 1998; 32: 588-601.

8. Dolovich LR, Ginsberg JS, Douketis JD, Holbrook AM, Cheah G. A meta-analysis comparing low-molecular-weight heparins with unfractionated heparin in the treatment of venous thromboembolism. Arch Intern Med 2000; 160: 181-88.

9. Gabriel Botella F, Labiós Gómez M, Balaguer Martínez JV, Fernández Llópez A. Prevención de la enfermedad tromboembólica venosa: heparinas no fraccionadas y heparinas de bajo peso molecular. Análisis de estas dos opciones. An Med Interna (Madrid) 1999; 16: 590-600.

10. Low molecular weight heparins for venous thromboembolism (rev). Drug Ther Bull 1998; 36: 25-9.

11. Fegan CD. Tinzaparin as an antithrombotic: an overview. Hosp Med 1998; 59: 145-8. 